\title{
Green IoT: Current Scenario \& Future Prospects
}

\author{
Malti Bansal ${ }^{1, *}$, Aayush Kumar ${ }^{1}$, Aman Virmani \\ ${ }^{1}$ Department of Electronics \& Communication Engineering, \\ Delhi Technological Univerity (DTU), Delhi-110042, India \\ ${ }^{2}$ Department of Electrical Engineering, \\ Delhi Technological Univerity (DTU), Delhi-110042, India \\ "maltibansal@gmail.com
}

\begin{abstract}
Demands of world's whole population for insubstantial products has thus prompted the need of effective utilization of materials and assets. As huge changes in society have been impacted for good by the advancement in Information and Communication Technology, their use has a negative effect on the environment and human wellbeing. The reason being, our society is in a deep need of a greener future where there is judicial use of resources along with minimal use of non-renewable resources. This will eventually decrease the pollution \& promote lesser power consumption. Green Internet of Things takes one of the main parts while in transit to make a sustainable \& green spot for living. We investigate and talk about how the different empowering advancements, (for example, the Internet, smart objects, sensors, and so forth) can be effectively implemented to accomplish a green IoT. Moreover, we likewise audit different IoT applications, standardization and projects which are at present under way. At last, we recognize a portion of the challenges which should be tended to later on to empower a green IoT.
\end{abstract}

Keywords—Green WSN, Green DC, Green RFID, Green IoT, Smart City, Energy Efficient, Future of IoT

\section{INTRODUCTION}

Our world is connected by means of worldwide communication networks utilizing (TCP/IP) convention which makes the world as a little town where various things are associated with one another. Specialized gadgets, yet additionally actual objects, similar to vehicles, PCs, and home appliances, which are controlled through remote communication networks are some of the things which are incorporated. There is an estimation that these connected devices will going to get hit 50 billion[1] by the end of 2020 and 75 billion by the end of 2025. With this increase in the number of devices, scientists also expect an increase in data rate and content size at cost of exceptional ' $\mathrm{C}$ ' emissions into the environment. Currently the Information and Communication Technology produces $6 \%$ of the global carbon footprint and due to emerging demand it is estimated that by 2030 this will double. Due to this tremendous expectation of increased $\mathrm{CO}_{2}$ emissions a green or renewable technology is gaining an attractive research area in the technology evolution. Shrewd availability of the current networks and setting mindful computation utilizing framework resources has become a significant piece of the internet of things.

\section{INTERNET OF THINGS AND GREEN IOT}

Physical objects are connected to the internet in a IoT based network with the web through network devices or routers and exchange of information is made. IoT permits objects to be controlled distantly across existing network infrastructure and is a generally excellent method which decreases human exertion just as makes it simple to get to actual gadgets. IoT can be categorized into three broad categories which is given below

- Hardware

- Software

- Cloud

In general, IoT hardware includes:

- Sensors

- Microcontrollers

- Communication modules

\section{- Hardware}

It mainly includes Sensors, Microcontrollers and communication modules. Sensors are developed from research in the field of material science. Sensors collect data and send data to microcontrollers which are 
Journal of trends in Computer Science and Smart technology (TCSST) (2020)

Vol.02/ No. 04

Pages: 173-180

https://www.irojournals.com/tcsst/

DOI: https://doi.org/10.36548/jtcsst.2020.4.001

essentially connected to the internet. Some examples of the sensors are photoelectric sensors, water quality sensors etc.

- Microcontrollers

Microcontrollers are basically the brains of all the systems, each sensor is connected to a microcontroller which then reads, analyzes, filters and sends the data to the master node.

\section{- Communication Modules}

Communication modules are like the nerves in a human body connecting everything to each other. Cloud includes fog computing which was introduced because of delay issues, now the microcontrollers are connected to a server where machine learning, data mining techniques are applied.

Furthermore, Basic elements in IoT are Things, Internet and Processing

- Internet

It provides communication between devices with the help of Internet Protocols. such as (SMTP) Simple Mail Transfer Protocol, (FTP) File Transfer Protocol, TCP/IP, (IMAP) Internet Message Access Protocol, (UDP) User Datagram Protocol, (POP3) Post Office Protocol, (HTTP) Hypertext Transfer Protocol. These protocols are widely used in applications of IoT.

- Processing

Processing of the data received by sensor or "Things" and producing a fruitful outcome which is done with the help of software and firmware which process the data with the help of microcontroller/ microprocessor

- Things

Things which act as a sensor, or an instrument which senses its surrounding, for instance, Radio-frequency identification (RFID) [2]. Things which acts as an actuator, or action performing component

\section{GREEN IoT}

Green IoT can be characterized as the energy proficient methodology inherited by IoT either to encourage decreasing the greenhouse effect of current applications and administrations or to lessen the effect of greenhouse effect of IoT itself. In the previous case, the utilization of IoT will help diminish the greenhouse effect, while in the later case further improvement of IoT greenhouse impression is to be taken consideration. IoT models are not optimized for energy efficiency; hence they waste energy because they are always on even during not required. Due to this plenty of energy is consumed while it on and sends data transmitting $24 * 7$.

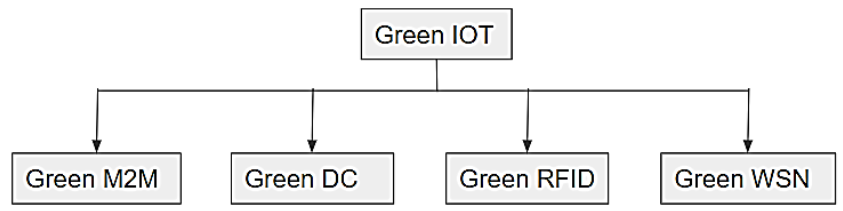

Fig. 1 Components of Green IoT

In Green IoT, it is ensured the device is on only when it is required and idle or off when not required. Green IoT focuses on the smart operation of devices with a decrease in energy waste. Proper Ventilation of Heat Generated from Servers and Data Centers, Conserve Energy using Smart IoT Technology The whole life pattern of green IoT centers around green plan, green creation, green usage, lastly green removal/reusing which effects the environment with no or little impact.

Green IoT mainly consists of communication technologies like Green RFID, Green WSN , Green cloud computing . Green M2M (Machine to Machine) \& Green Data Centres. They mainly require special hardware and software which are specially designed to consume less energy without reducing performance while minimizing utilization resources.

\section{Green RFID Tags}

RFID stands for radio frequency identification and green RFID [3] tags store data at a small level for the sensors they are linked with. RFID tags are two types i.e. Active tags which have built in batteries for 
continuous transmission of their own signal and the other type is passive tags which do not have an active battery source. They store energy from the reader. A proper optimisation in use of the RFID tags can help in decreasing the energy consumption as well as reduce the amount of non-degradable material

\section{Green Wireless Sensor Network}

Green WSN [4] is based on Green Energy Conservation technique, Radio Optimization technique, Green routing techniques that all lead to reduction in energy consumption. WSN consists of minimum required sensors with the limited power and storage capacity. Also smart data algorithms which reduces storage capacity requirements as well data size are used also a smarter approach is used to activate sensors when required

\section{Green DC}

Data centers are like the information houses in IoT which spread almost all types of data as well as applications [5]. Their task is simple to store, manage and to process the data. Apart from these routing protocols, in these data centers are designed to switch off the idle devices hence making the protocols energy efficient and environment friendly.

\section{Green Machine to Machine Technology}

Recently, the machines in the modern world are becoming smarter and gathering data with no human exertion or intervention. A.I. Artificial Intelligence is the cause after all this, and to evolve more the machines need to constantly talk to each other. This requires a good connectivity and also a huge bandwidth hence leading to more energy consumption and pollution. Optimized connectivity techniques have been opted in the method to reduce the carbon footprint [6].

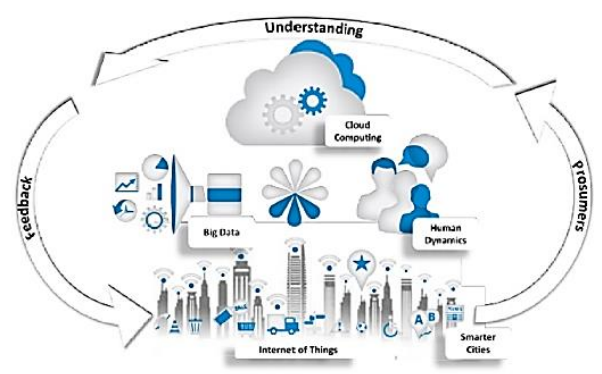

Fig. 2 Ecosystem of Green IoT

\section{APPLICATIONS OF GREEN IOT}

Noteworthy alteration in this current environment have occurred, and further alteration would happen soon on behalf IoT advancements. Be that as it may, improvement costs are critical because of expanded hazardous emissions, e-waste \& power consumption. As Green IoT assessed to have a major effect in our forthcoming lives $\&$ could prompt a greener space. Ofcourse in coming future, we would find in our day by day lives numerous devices, industrial machines, sensors, drones, and functional objects communicate to achieve, with their insight, their assignments for the green climate. Subsequently, green IoT applications center around energy preservation, lessening $\mathrm{CO} 2$ emanations and harmful pollution. Nonetheless exclusively green IoT is assisting different businesses with lessening the impact of a worldwide temperature alteration yet it additionally diminishes the effect of IoT itself on the climate. This helps IoT in investigating diverse, environment friendly fuel sources, lessening the IoT generated risk in the climate. Therefore, widespread use of green IoT has a reason, reserve funds, climate and social supportability, and to conserve cnatural resources and improve wellbeing of human.

\section{Smarter Home :}

The green IoT makes home warming, lighting, and appliances controlled by a computer / smartphone. The cell phone / home computer receives voice commands. Lighting creates unnatural or natural solar flares, rooms and windows equipped with insulated soundproof electric equipments [7]. Recognizes occupants with customized activities and reactions, Television, PC, and telephone joined into one gadget, and so forth. Life cycle of green IoT must be considered consisting of green formations, green materials, green production, and green waste disposal / reuse; reducing the impact on the environment.

\section{Industrial Automation :}

Industries are made up of machines that will be able to do the job well without the slightest manual-based intervention. 
a. Machine-to-machine communication: by using RFID automation tags it is also possible for an RFID reader to speak directly to a assistant involving no human exertion.

b. Monitoring Plant: Emerging advancements can adequately screen different boundaries, for example, mechanical issues, temperature, air contamination, and so on, of modern plant in order to improve effectiveness of energy.

\section{Smart Health care :}

In the human body, the use of biometric sensors and actuators in patients with gripping, tracking and monitoring [8]. The deliveries of effective health care services improve the care quality, improve admittance to mind, \& diminish the care expense.

a. Real Time Tracking: This involves, Monitoring \& Tracking of patients \& equipment of medical care. On behalf of terms of goods, following \& tracking helps the repairs, detection, use of monitoring, \& objects tracking for preventing various metal unknowingly to be left on the body of the patient during surgery. [9]

b. Identification: Power proficiency accomplished by implementing viable techniques of track, successful RFID tracking, predictive strategies, and so on. These are important to get patient details quickly and to monitor areas in the emergency clinic of the patients. Advancements like these must uphold understanding recognizable proof to diminish quiet risk factors, (for example, drug misuse, dose, and so on), electronic wellbeing records (inside \& outside the patient), \& pediatric ID (to forestall misdiagnosis).

c. Advancement in Collection of Data: decreases preparing time, measure mechanized hospitalization, and computerized care \& cycle assessment, and lessens costs.

d. Sensing in smart way: Neurological gadgets give persistent demonstrative conditions and ongoing wellbeing pointers.

\section{Smarter Grid :}

Consciousness of public in the swapping worldview of intensity supply, utilization, \& foundation right on growing. Rather than depending on petroleum derivatives, atomic energy, needs of the future energy should be founded fundamentally on an assortment of sustainable assets. Power of future grid ought to handily respond to control changes in power, fuel sources and consumer utilization. This type of a grid would get founded as the collection of associated network shrewd gadgets (hardware, assembling, infrastructure, and customer items) in view of IoT ideas.

\section{Smart Cities :}

Characterization of IoT should be done by utilization of active energy to empower a sustainable world [10]. Therefore, in order to change world for more smartness various machinery being undergone so as to customize them with extra sensors and interchanges to make the world more astute. The communication can be done by this in a smarter way by sensing and detecting encompassing objects and exchange data by one another in the city. This concept of a smarter city incorporates smart parking [11], bright keen lights [12], top notch air with better AQI, smart vehicle [13], smart execution of traffic [14], smart buildings, garbage collection, water sensors, smart metering and more.

\section{Smarter Environment :}

The expanding count in vehicles, metropolitan relocation \& numerous modern exercises have significantly expanded AQ contamination over the most recent couple of many years. Observing contamination of air is viewed as the most intricate and significant task. Utilization of IoT innovation may happen to undergo air contamination checking least mind boggling \& help to more readily comprehend the climate [16], For reduction in the natural effect of waste disposal, numerous organizations \& municipal structures are associated with the improvement of effective garbage administration frameworks. Putting RFID sensors on the trash bin makes them more smarter. At the point when trash (with the RFID tag) is set in a receptacle, the container can distinguish the sort of waste to simplify the reusing cycle [17], helping agribusiness: this is going to empower farmers so they can address significant difficulties they face. The business should take a gander at available 


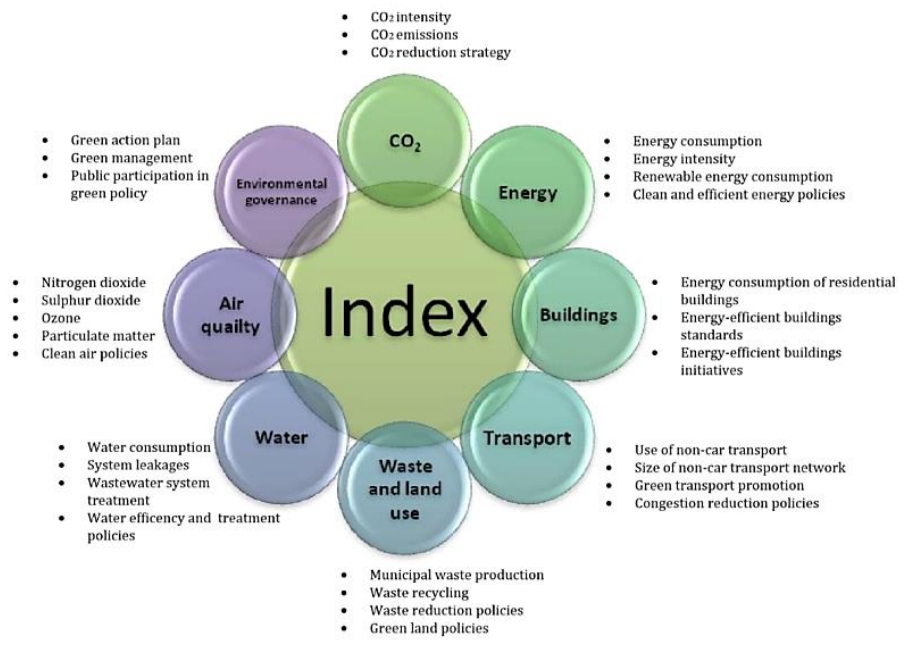

Fig.3: Ecosystem of Green IoT

resources so water scarcity can be addressed, expenses management, \& at last restricted land accessibility. Green IoT \& green nanotechnology arise by fulfilling answers for sake of feasible agriculture \& food industry to be more smarter[15]. Such smart practices help promote a healthy, sustainable and future proof environment.

\section{FUTURE DIRECTIONS OF GREEN IOT}

Green IoT can change our vivid future tomorrow to be ambitious and greener, higher Quality of Service, socially and ecologically sustainable. Today, the highly exciting places center around green things like green networking \& communication, green IoT plan \& usage, power decreasing methodologies, integrated RFID \& sensory networking, network management \& mobility, collaborative and diverse network collaboration, smart things, and performance of the green area. The following areas of research required research to find sound and effective greening IoT solutions:

1. An enormous amount of IoT gadgets should be supplanted with UAV, particularly in horticulture, roads \& surveillance, helping in diminishing energy utilization \& air contamination. An arising innovation 'UAV' will prompt green IoT with ease and high productivity.

2. Information movement from sensor to cellular cloud has been valuable. Cloud - sensor incorporates cell cloud \& (WSN) wireless sensor network. It is a splendid and promising innovation for green IoT. Green communication network for example, a help (SNaaS) can investigate system performance, service, cloud management and WSN.

3. M2M connectivity plays a very important role in reducing energy consumption, dangerous emissions. Smart machine should be smart enough so that systems can be made automated enabled. In the event of traffic, programmed systems delays should be reduced and immediate \& necessary steps should be taken.

4. Design of Green IoT should get imported through targets that achieve high Quality of Service and excellent performance. Finding the right strategies to improve Quality of Service framework (e.g., Bandwidth, delays, $\&$ installations) will pump up the effectiveness and efficiency in the expansion of IoT.

5. Moving forward in the direction of green IoT, less amount of energy is consumed, demand of newer assets $\&$ resources, limiting IoT negative effect on the soundness of human and upsetting the climate. At that point contribution of green IoT can fundamentally help to build a maintainable shrewd and green climate. 
Journal of trends in Computer Science and Smart technology (TCSST) (2020)

Vol.02/ No. 04

Pages: 173-180

https://www.irojournals.com/tesst/

DOI: https://doi.org/10.36548/jtcsst.2020.4.001

6. To accomplish a power balance that facilitates green communication among IoT devices, the reaping intensity of radio waves must be thought of.

7. There is much research requirement to improve the design of IoT devices that help in reduction of $\mathrm{CO}_{2}$ emissions \& energy consumption. The most important function of a healthy and green environment is to conserve energy and reduce the $\mathrm{CO} 2$ emissions.

\section{RELATED STANDARDIZATION \& PROJECTS}

Governments and various organizations across world pushing themselves in making efforts \& performing a significant involvement in uplifting society through the involvement of different Governments around globe and making efforts in making companies for minimizing emissions of $\mathrm{CO}_{2}$, evolve in a way greater energy efficient, and adopt more greener ways.

There is a project named TREND[18], it basically fetches data of energy consumption, tests technologies that claims to save energy, agreements, new architectures, and tests in afresh ways. Also, conducts training programs for spreading awareness of the GreenNet (a green network).

Another Project, Project EARTH[19] which investigates how much energy efficient wireless communication network are. Mainly focused around concept as well as the effective boundary of existing networks which presumed to build a newer generations of equipment which will be more energy efficient, operating techniques, and managing network for solutions in order for ensurity of service quality.

A Subcommittee on Agricultural \& Computer Communication Technology (TSCGCC) is also established by the Society of IEEE Communication [20]. The TSCGCC processes around to build, balance power and obviously the power connections. Certianly accords opportunities for communication \& interchange of technological plans, identifying R\&D confrontation, and collaborating over results to develop most powerful communications and computer technology in the environment.

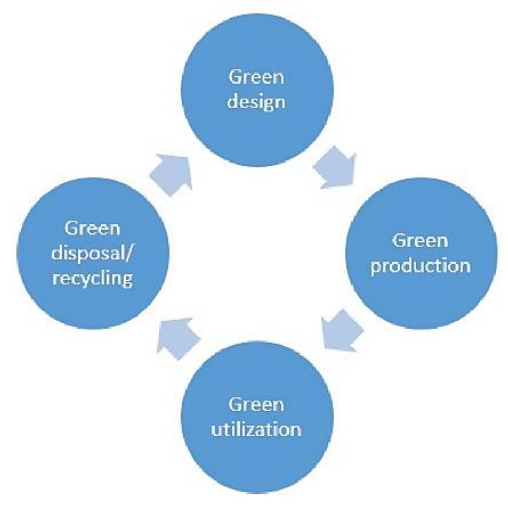

Fig. 4: Green IoT Cycle

\section{ISSUES AND CHALLENGES}

The main principle role of Green technologies would be in providing IoT in a manner which is efficient on the terms of energy. Today, there exist a lot of problems which must be dealt with. Provided are the prominent issues which infer that they require more thinking [21-26].

\section{Architecture:}

For IoT, widely known architectures which are TCP / IP model or ISO / OSI model are necessary to be implemented in order occupy varied type devices that have diverse applications and heterogeneous networks to establish a communication across them, there is a need for understanding so that to collect energy efficient connection for all structures to achieve more energy performance.

\section{Infrastructure:}

IoT energy efficiency infrastructure can be accessed through the review process. 
Journal of trends in Computer Science and Smart technology (TCSST) (2020)

Vol.02/ No. 04

Pages: 173-180

https://www.irojournals.com/tcsst/

DOI: https://doi.org/10.36548/jtcsst.2020.4.001

\section{Management of Spectrum:}

Comprehension of radio framework carries numerous advantages to the green cloud services majority of which are right now restricted to rf programs.

\section{Energy-saving communications:}

Energy-saving communications face numerous moves, for example, giving consistent power to loop objects and holding up energy-proficient communication agreements so that reliable communication can be enabled among the peers, etc. Good reception of new sources of energy, for example, wind, sun, heat, \& vibration in order to promise current of scope green iot to be more reliable.

\section{Providing QoS:}

Privacy protection \& Security are significant worries of IoT exports. Using security-algorithms requires a large amount of processing from devices.

In spite of the fact that there exists a great deal of exploration papers on IoT frameworks, analysts, developers are as yet zeroing in on distinguishing the energy hungry parts/angles in IoT frameworks \& are chipping away at advancement arrangements.

\section{CONCLUSION}

From a human standpoint, the tendency to have a safe, comfortable, and stable environment has not changed. With the advancement of technology, the idea of smarter, safer and greener living spaces becomes a reality. The growing population and reduction in urban areas means an increase in the use of limited resources and resources, as well as waste and increased emissions. While new technologies play a key role in achieving a smart and sustainable view of the city, they at the same time have a devastating effect on the environment and for this in the human race. To address these difficult confrontations, there is somewhat need of necessary action to take, that going to minimize the human well-being \& environment's negative impacts. Moving towards greener technology, specifically Green IoT, seems like a reasonable step. The design, production, use and disposal of raw materials and materials (without adversely affecting environment $\&$ the human health) make Green IoT the pillar of the most intelligent \& technologically advanced evolution in the world. The vision of a smart city leads to the creation of a volume and various details that are produced on a daily basis by installation of Green IoT related services \& devices. Future proposals have been made to effectively and efficiently develop green programs for IoT. This study provides an effective understanding for anyone wishing to pursue insight in the field of green IoT.

\section{REFERENCES}

[1] H. S. Dunn, "The carbon footprint of ICTS," Global Inf. Soc. Watch, Univ. West Indies, Kingston, Jamaica, 2010.

[2] David L. Brock, "The electronic product code (epc)." Auto-ID Center White Paper MIT-AUTOID-WH-002 (2001)

[3] Namboodiri, Vinod, and Lixin Gao. "Energy-aware tag anticollision protocols for RFID systems." IEEE Transactions on Mobile Computing 9.1 (2010): 44-59.

[4] Lin, Yu-Hsiang, et al. "Optimal and maximized configurable power saving protocols for corona-based wireless sensor networks." IEEE Transactions on Mobile computing 14.12 (2015): 2544-2559.

[5] Chang, Chih-Hua, Ronald Y. Chang, and Hung-Yun Hsieh. "Highfidelity energy-efficient machine-tomachine communication. " Personal, Indoor, and Mobile Radio Communication (PIMRC), 2014 IEEE 25th Annual International Symposium on. IEEE, 2014.

[6] Shuja, Junaid, et al. "Survey of techniques and architectures for designing energy-efficient data centers." IEEE Systems Journal 10.2 (2016): 507-519.

[7] M. Elkhodr, S. Shahrestani and H. Cheung,"The Internet of Things: Vision \& Challenges," 2013 IEEE Tencon, pp. $218-222,2013$.

[8] Z.M. Kalarthi, A review paper on smart health care system using Internet of Things, International Journal of Research in Engineering and Technology (IJRET), 5 (2016) 80-83.

[9] It's Official: The Internet Of Things Takes Over Big Data As The Most Hyped Technology, [online] Available: online

[10] P. Sathyamoorthy, E.C.-H. Ngai, X. Hu, V.C. Leung, Energy efficiency as an orchestration service for mobile Internet of Things, Cloud Computing Technology and Science (CloudCom), 2015 IEEE 7th International Conference on, IEEE, 2015, pp. 155-162. 
Journal of trends in Computer Science and Smart technology (TCSST) (2020)

Vol.02/ No. 04

Pages: 173-180

https://www.irojournals.com/tcsst/

DOI: https://doi.org/10.36548/jtcsst.2020.4.001

[11] P. Ramaswamy, Iot smart parking system for reducing green house gas emission, Recent Trends in Information Technology (ICRTIT), 2016 International Conference on, IEEE, 2016, pp. 1-6.

[12] M. Popa, A. Marcu, A Solution for street lighting in smart cities, Carpathian Journal of Electronic and Computer Engineering, 5 (2012) 91.

[13] A.M. Vegni, M. Biagi, R. Cusani, Smart vehicles, technologies and main applications in vehicular ad hoc networks, Vehicular Technologies-Deployment and Applications, InTech, 2013.

[14] K. Su, J. Li, H. Fu, Smart city and the applications, Electronics, Communications and Control (ICECC), 2011 International Conference on, IEEE, 2011, pp. 1028-1031.

[15] M. Maksiimovici, E. Omanovic-Miklicanin, Green Internet of Things and Green Nanotechnology Role in Realizing Smart and Sustainavle Agriculture VIII International Scientific Agriculture Symposium "AGROSYM 2017", Jahorina, Bosnia and Herzegovina, 2017.

[16] K.K.Khedo, R.Perseedoss, andA.Mungur,“Awireless sensornetwork air pollution monitoring system,” Int. J. Wireless Mobile Netw., vol. 2, no. 2, pp. 31-45, May 2010

[17] Faisal Karim Shaikh, Member, IEEE, Sherali Zeadally, Senior Member, IEEE, and Ernesto Exposit.

[18] TREND, Accessed Feb. 20, 2014. [Online]. Available: www.fp7-trend. eu

[19] EARTH, Accessed Feb. 20, 2014. [Online]. Available: https://www.ictearth.eu/

[20] Green Comm. \& Comp. [Online]. Available: www.comsoc.org/about/ committees/emerging\#gcc

[21] The Internet of Things (IoT): An Overview, Understanding the issues of more connected world.Internet Society.

[22] M. Bansal and Priya, "Application Layer Protocols for Internet of Healthcare Things (IoHT)," 2020 Fourth International Conference on Inventive Systems and Control (ICISC), Coimbatore, India, 2020, pp. 369-376, doi: 10.1109/ICISC47916.2020.9171092.

[23] M. Bansal, Priya, "Performance Comparison of MQTT and CoAP Protocols in Different Simulation Environments". G. Ranganathan, J. Chen, A. Rocha (eds) Inventive Communication and Computational Technologies. Lecture Notes in Networks and Systems, vol. 145, pp. 549-560, Springer, Singapore, https://doi.org/10.1007/978-981-15-7345-3_47

[24] M.Bansal, Priya, "Machine Learning Perspective in VLSI Computer Aided Design at Different Abstraction Levels", $2^{\text {nd }}$ Int. Conf. on Mobile Computing and Sustainable Informatics (ICMCSI 2021), Tribhuvan University, Nepal, 2021.

[25] M.Bansal, A. Goyal, A. Choudhary, "Industrial Internet of Things (IIoT): A Vivid Perspective", $5^{\text {th }}$ Int. Conf. on Inventive Systems and Control (ICISC 2021), India, 2021.

[26] M. Bansal, V. Sirpal, M. K. Choudhary, "Advancing e-Government using Internet of Things", ”, 2 nd Int. Conf. on Mobile Computing and Sustainable Informatics (ICMCSI 2021), Tribhuvan University, Nepal, 2021. 